\title{
ESTABILIDADE DE PRODUÇÃO DE CULTIVARES DE FEIJÃO DE DIFERENTES GRUPOS COMERCIAIS NO ESTADO DO RIO GRANDE DO SUL( $\left.{ }^{1}\right)$
}

\author{
NERINÉIA DALFOLLO RIBEIRO $\left({ }^{*}\right)$; JUAREZ FERNANDES DE SOUZA $\left({ }^{3}\right)$; \\ IRAJÁ FERREIRA ANTUNES $\left({ }^{4}\right)$; NERISON LUIS POERSCH $\left({ }^{5}\right)$
}

\begin{abstract}
RESUMO
O objetivo deste trabalho foi avaliar a adaptação e a estabilidade de produção de cultivares de feijão para o cultivo no Estado do Rio Grande do Sul. Assim, 16 cultivares de diferentes grupos comerciais foram avaliadas em 20 ambientes, no Estado, durante os anos agrícolas de 2004/2005 a 2006/2007. As estimativas de adaptabilidade e de estabilidade foram obtidas pelos métodos de Eberhart e Russel e de Lin e Binns, modificado por Carneiro. Interação cultivares com ambientes significativa foi observada para a produtividade de grãos, indicando resposta diferenciada das cultivares aos locais e anos avaliados. Os resultados evidenciaram que as metodologias foram concordantes na identificação de cultivares estáveis para produtividade de grãos (Guapo Brilhante) e com adaptação a ambientes favoráveis (BRS Expedito e TPS Bonito). A cultivar Guapo Brilhante é de alta produtividade média de grãos, com adaptação e estabilidade de produção para o cultivo no Estado do Rio Grande do Sul.
\end{abstract}

Palavras-chave: Phaseolus vulgaris L., interação genótipos com ambientes, rendimento de grãos, adaptabilidade, estabilidade.

\section{ABSTRACT \\ YIELD STABILITY OF COMMON BEAN CULTIVARS OF DIFFERENT COMMERCIAL GROUPS IN RIO GRANDE DO SUL STATE}

The objective of this research was to evaluate the adaptability and yield stability of common bean cultivars for the cultivation in the Rio Grande do Sul State. Sixteen common bean cultivars of different commercial groups were evaluated in three growing seasons, in 20 environments, in the Rio Grande do Sul State. The adaptability and stability estimates were obtained for the methods of Eberhart and Russel and Lin and Binns, modified to Carneiro. Significative cultivars x environments interaction were observed for grain yield indicating different responses of the cultivars to the assayed locations and years. The results showed that the two methods gave similar results in the identification of cultivars with yield stability (Guapo Brilhante) and with adaptability to favorable environment (BRS Expedito e TPS Bonito). The Guapo Brilhante cultivar showed high grain yield, with adaptability and yield stability for cultivation in the Rio Grande do Sul State.

Key words: Phaseolus vulgaris L., genotype $\mathrm{x}$ environment interaction, grain yield, adaptability, stability.

${ }^{1}$ ) Recebido para publicação em 5 de março de 2008 e aceito em 11 de fevereiro de 2009.

$\left({ }^{2}\right)$ Departamento de Fitotencia, Centro de Ciências Rurais, Universidade Federal de Santa Maria (UFSM), 97105-900 Santa Maria (RS). E-mail: neiadr@smail.ufsm.br (*) Autora correspondente.

$\left({ }^{3}\right)$ Fundação Estadual de Pesquisa Agropecuária (FEPAGRO), 90130-060 Porto Alegre (RS). E-mail: juarez-souza@fepagro.rs.gov.br

$\left({ }^{4}\right)$ EMPRAPA Clima Temperado, Caixa Postal 553, 96001-000 Pelotas (RS). E-mail: iraja@cpact.embrapa.br

$\left({ }^{5}\right)$ Aluno do Programa de Pós-graduação em Agronomia da UFSM. 


\section{INTRODUÇÃO}

O feijão (Phaseolus vulgaris L.) possui teores significativos de proteínas, carboidratos, vitaminas, minerais e fibra, baixo conteúdo de gordura e de colesterol (Hosfield, 1991; Morrow, 1991), por isso é considerado de alta qualidade nutricional e funcional. Além disso, por razões culturais e econômicas, é amplamente utilizado na alimentação, independentemente da classe social (ANTUNES et al., 1995). É a principal fonte de proteína da população de menor poder aquisitivo.

O Brasil é um dos maiores consumidores per capita dessa leguminosa. Entretanto, a produtividade média nacional é de $817 \mathrm{~kg} \mathrm{ha}^{-1}$ e no Estado do Rio Grande Sul, $1223 \mathrm{~kg} \mathrm{ha}^{-1}$ (CONAB, 2007). A baixa produção é conseqüência do baixo nível tecnológico e da diversidade de condições ambientais em que o feijão é cultivado. Assim, diferenças entre locais, anos e épocas de avaliação têm sido observadas em diversas regiões do país (ABREU et al., 1998; COIMBRA et al., 1999; NunEs et al., 1999; PIANA et al., 1999; BORGES et al., 2000; CARBONELl et al., 2001; RibEIRO et al., 2004; BACKes et al., 2005; CARbonell et al., 2007; Melo et al., 2007).

Considerando a necessidade de identificar cultivares de feijão com ampla adaptabilidade e maior estabilidade fenotípica, como alternativa para atenuar os efeitos da interação genótipos com ambiente, decidiu-se através da Comissão Estadual de Pesquisa de Feijão do Rio Grande do Sul (CEPEF) desenvolver em rede os experimentos de avaliação de cultivares. Dessa maneira, os programas públicos de melhoramento de feijão do Rio Grande do Sul Embrapa Clima Temperado, Universidade Federal de Santa Maria (UFSM) e Fundação Estadual de Pesquisa Agropecuária (FEPAGRO) - avaliam de maneira conjunta as cultivares de feijão inscritas no Registro Nacional de Cultivares, no Ministério da Agricultura (RNC-MAPA) e aquelas tradicionalmente em uso pelos pequenos produtores rurais, com a finalidade de gerar informações sobre adaptação varietal às condições de cultivo do Rio Grande do Sul.

Nos últimos anos, vários métodos foram desenvolvidos para identificar cultivares com alto desempenho médio nos vários ambientes de cultivo (Cruz e Regazzi, 2001; Cruz e Carneiro, 2003). Na cultura do feijão, as metodologias de Eberhart e Russel e de Lin e Binns têm sido as mais utilizadas (AвREU et al., 1998; CoImbra et al., 1999; Nunes et al., 1999; PiAna et al., 1999; Borges et al., 2000; CARBonell et al., 2001; RibeIRO et al., 2004; BACKES et al., 2005; CARBONELL et al., 2007; Melo et al., 2007), visto que esses métodos são de simples interpretação, pois agregam adaptabilidade, estabilidade e produtividade em uma única estatística. Pelo método de Eberhart e Russel (1966), a cultivar ideal é aquela em que se constata coeficiente de regressão igual à unidade $\left(\beta_{\mathrm{i}}=1\right)$, desvio de regressão o menor possível $\left(\mathrm{s}^{2}{ }_{\mathrm{d}}=0\right)$ e alta produtividade média. Por sua vez, o método de LiN e BINNs (1988) mede a estabilidade pela estimativa do índice de superioridade $P_{i}$. Assim, são desejáveis as cultivares com menor $P_{i}$, pois ocorre menor desvio em relação à produtividade máxima em cada ambiente, isto é, tem desempenho próximo do máximo na maioria dos ambientes. Além disso, esses métodos fornecem informações complementares (SILVA e DuARte, 2006; Melo et al., 2007).

CARNeIro (1998) propôs uma modificação na metodologia de Lin e Binns, ou seja, fez a decomposição do estimador $P_{i}$ em duas partes $P_{\text {if }}$ (ambientes favoráveis) e em $P_{\text {id }}$ (ambientes desfavoráveis). Dessa maneira, foi possível identificar germoplasma de feijão com maior estabilidade $\left(<\mathrm{P}_{\mathrm{i}}\right)$, mais responsivos a ambientes favoráveis $\left(<\mathrm{P}_{\mathrm{if}}\right)$ e mais adaptados a ambientes desfavoráveis $\left(<\mathrm{P}_{\mathrm{id}}\right)$ (CARbonell et al., 2001; Melo et al., 2007).

Assim, o objetivo deste trabalho foi avaliar a adaptação e a estabilidade de produção de cultivares de feijão, com a finalidade de identificação de cultivares mais estáveis e produtivas para o cultivo no Estado do Rio Grande do Sul.

\section{MATERIAL E MÉTODOS}

Os experimentos foram desenvolvidos nos anos agrícolas de 2004/2005, 2005 / 2006 e 2006/2007, em 10 municípios do Estado do Rio Grande do Sul: Frederico Westphalen $\left(53^{\circ} 23^{\prime} \mathrm{W}-27^{\circ} 21^{\prime} \mathrm{S}\right)$, Pelotas $\left(52^{\circ} 10^{\prime} \mathrm{W}-31^{\circ} 70^{\prime} \mathrm{S}\right)$, Júlio de Castilhos $\left(53^{\circ} 40^{\prime} \mathrm{W}\right.$ $\left.29^{\circ} 13^{\prime} \mathrm{S}\right)$, Santo Augusto $\left(53^{\circ} 46^{\prime} \mathrm{W}-27^{\circ} 51^{\prime} \mathrm{S}\right)$, Maquiné $\left(50^{\circ} 12^{\prime} \mathrm{W}-2^{\circ} 40^{\prime} \mathrm{S}\right)$, Santa Maria $\left(53^{\circ} 43^{\prime} \mathrm{W}-2^{\circ} 42^{\prime} \mathrm{S}\right)$, São Borja $\left(56^{\circ} 00^{\prime} \mathrm{W}-28^{\circ} 39^{\prime} \mathrm{S}\right)$, Palmeira das Missões $\left(53^{\circ} 18^{\prime} \mathrm{W}-27^{\circ} 53^{\prime} \mathrm{S}\right)$, Vacaria $\left(50^{\circ} 56^{\prime} \mathrm{W}-28^{\circ} 30^{\prime} \mathrm{S}\right)$ e Veranópolis $\left(51^{\circ} 31^{\prime} \mathrm{W}-28^{\circ} 56^{\prime} \mathrm{S}\right)$. A semeadura foi realizada em setembro-outubro (cultivo de safra), exceto em São Borja que foi em janeiro (cultivo de safrinha), totalizando 20 ambientes (Tabela 1). Os experimentos de Frederico Westphalen e de Pelotas foram coordenados pela Embrapa Clima Temperado. A Universidade Federal de Santa Maria, por sua vez, foi responsável pelo experimento de Santa Maria e a FEPAGRO gerenciou os demais.

Um total de 21 cultivares de feijão foram avaliadas, sendo 15 do grupo comercial preto (Rio Tibagi, Guateian 6662, Macanudo, Minuano, Macotaço, IAPAR 44, Guapo Brilhante, TPS Nobre, Diamante Negro, BRS Valente, FTS Soberano, BRS Expedito, TPS Bionobre, BRS Campeiro e FEPAGRO 
26), cinco cultivares com grãos tipo carioca (Carioca, Pérola, FTS Magnífico, TPS Bonito e IAPAR 31) e uma, com grãos tipo manteigão (Iraí). As cultivares Rio Tibagi, Guateian 6662, Macanudo, Minuano, Macotaço, Guapo Brilhante, Diamante Negro, BRS Valente, FTS Soberano, BRS Campeiro, FEPAGRO 26, Pérola, FTS Magnífico e Iraí são indicadas para o cultivo no Rio Grande do Sul, de acordo com o Zoneamento Agrícola de Risco Climático (MAPA, 2007) e, por isso, foram consideradas cultivares testemunhas. As cultivares IAPAR 44, TPS Nobre, Carioca, TPS Bonito, IAPAR 31 e TPS Bionobre não estavam registradas no MAPA, mas foram incluídas porque apresentavam área de cultivo representativa nas pequenas propriedades agrícolas gaúchas. A cultivar BRS Expedito foi avaliada, pois havia a possibilidade de sua inclusão no RNC-MAPA para o cultivo no Rio Grande do Sul.

O delineamento experimental utilizado foi o de blocos ao acaso, com três repetições. A unidade experimental foi composta por duas fileiras de $4 \mathrm{~m}$ de comprimento, espaçadas de $0,5 \mathrm{~m}$, e área útil de $4 \mathrm{~m}^{2}$. A densidade de semeadura foi ajustada de acordo com o hábito de crescimento de cada cultivar (CEPEF, 2003).

O solo foi preparado de maneira convencional e a adubação foi realizada de acordo com a interpretação da análise química do solo. O controle de insetos foi efetuado com a aplicação de Metamidofós e o controle de plantas invasoras foi manual e efetuado sempre que necessário, de maneira que não houvesse competição com a cultura. $O$ controle de doenças não foi realizado.

A colheita e a trilha das plantas foram realizadas manualmente na maturação fisiológica. Após a retirada das impurezas, os grãos foram secados ao sol ou em estufa $\left(65\right.$ a $\left.70^{\circ} \mathrm{C}\right)$, até umidade média de $13 \%$, quando se determinou o rendimento de grãos.

Tabela 1. Número de cultivares avaliadas, produtividade de grãos, quadrado médio do erro (QM do erro) e coeficiente de variação (CV\%) em cada um dos 20 ambientes

\begin{tabular}{|c|c|c|c|c|}
\hline $\begin{array}{l}\text { Ambiente } \\
\text { Ano }\left({ }^{1}\right) \text { - Local }\left({ }^{2}\right)\end{array}$ & Cultivares & Produtividade & QM do erro & $\mathrm{CV}$ \\
\hline & $\mathrm{N}^{\circ}$ & $\mathrm{kg} \mathrm{ha}^{-1}$ & & $\%$ \\
\hline $1-1$ & 19 & 3079 & 486889,42 & 22,66 \\
\hline $1-2$ & 19 & 2153 & 40697,48 & 9,37 \\
\hline $1-3$ & 17 & 307 & 15067,37 & 39,97 \\
\hline $1-4$ & 17 & 1006 & 9385,19 & 9,62 \\
\hline $1-5$ & 20 & 1346 & 19416,46 & 10,35 \\
\hline $1-6$ & 18 & 1969 & 31189,15 & 8,97 \\
\hline $1-7$ & 17 & 1142 & 33515,76 & 16,02 \\
\hline $2-1$ & 20 & 1479 & 78091,37 & 18,89 \\
\hline $2-2$ & 20 & 2376 & 245051,27 & 20,84 \\
\hline $2-3$ & 19 & 637 & 19079,83 & 21,69 \\
\hline $2-4$ & 19 & 2074 & 31484,45 & 8,55 \\
\hline $2-5$ & 19 & 1996 & 135840,03 & 18,46 \\
\hline $2-6$ & 21 & 2351 & 165268,84 & 17,29 \\
\hline $3-2$ & 21 & 1360 & 216717,15 & 34,24 \\
\hline $3-4$ & 19 & 1598 & 57276,44 & 14,98 \\
\hline $3-5$ & 19 & 2798 & 229624,22 & 17,13 \\
\hline $3-6$ & 19 & 1352 & 115178,48 & 25,11 \\
\hline $3-8$ & 19 & 1623 & 23993,74 & 9,55 \\
\hline $3-9$ & 19 & 2066 & 64098,96 & 12,26 \\
\hline $3-10$ & 19 & 3256 & 182072,51 & 13,11 \\
\hline
\end{tabular}

( $\left.{ }^{1}\right)$ Ano agrícola: 1- 2004/2005; 2- 2005/2006; 3- 2006/2007.

$\left({ }^{2}\right)$ Local: 1- Frederico Westphalen; 2- Pelotas; 3- Júlio de Castilhos; 4- Santo Augusto; 5- Maquiné; 6- Santa Maria; 7- São Borja; 8- Palmeira, 9- Vacaria; 10- Veranópolis. 
Os dados obtidos foram submetidos à análise de variância individual e conjunta, considerando o efeito de cultivares como fixo e os efeitos de ambientes e de blocos, aleatórios. A análise conjunta foi realizada para as 16 cultivares comuns a todos os experimentos: Rio Tibagi, Guateian 6662, Macanudo, Minuano, Macotaço, IAPAR 44, Guapo Brilhante, TPS Nobre, Diamante Negro, BRS Valente, FTS Soberano, BRS Expedito, Carioca, Iraí, Pérola e TPS Bonito. O número de cultivares não foi constante nos diferentes experimentos, em razão da falta de sementes. $O$ índice ambiental foi calculado pela diferença entre a média das cultivares comuns em cada ambiente e a média geral das cultivares comuns em todos os ambientes.

As análises de adaptabilidade e de estabilidade foram realizadas de acordo com os métodos de Eberhart e Russell (1966) e de Lin e BinNs (1988), modificado por CARneIro (1998). Os testes estatísticos de Barlett e F máximo (Stell et al., 1997; Gomes, 1990; Cruz e Regazzi, 2001) foram aplicados para verificar a homogeneidade das variâncias residuais. As análises estatísticas foram processadas no programa GENES (CRUZ, 2006) e no aplicativo Office Excel.

\section{RESULTADOS E DISCUSSÃO}

A análise de variância individual foi realizada com todos os resultados verificados em cada ambiente, inclusive com aquelas cultivares que não foram consideradas para a análise conjunta (Tabela 1). Assim, observou-se que a produtividade média variou de $307 \mathrm{~kg} \mathrm{ha}^{-1}$ (Júlio de Castilhos, 2004/2005) a 3256 $\mathrm{kg} \mathrm{ha}{ }^{-1}$ (Veranópolis, 2006/2007). Apenas os experimentos realizados em Júlio de Castilhos, nos anos agrícolas de 2004/2005 e 2005/2006, tiveram produtividade média extremamente baixa, respectivamente, de $307 \mathrm{~kg} \mathrm{ha}^{-1}$ e $637 \mathrm{~kg} \mathrm{ha}^{-1}$, decorrente da deficiência hídrica registrada. Nos demais ambientes foi observada produtividade de grãos superior à média nacional de $817 \mathrm{~kg} \mathrm{ha}^{-1}$ (CONAB, 2007).

Os maiores coeficientes de variação foram constatados nos experimentos de Júlio de Castilhos - 2004/2005 (39,97\%) e de Pelotas - 2006/2007 $(34,24 \%)$. Somente nesses dois ambientes houve baixa precisão experimental e, de acordo com a instrução normativa número 25 , de 23 de maio de 2006, não poderiam ser aproveitados para a inscrição de cultivares de feijão no RNC-MAPA, pois excederam o valor máximo de $25 \%$ que pode ser considerado para o coeficiente de variação (BRASIL, 2006).

A relação entre o maior e o menor quadrado médio do erro $\left(\mathrm{QMr}^{+} / \mathrm{QMr}^{-}\right)$, da análise de variância individual dos 20 experimentos, foi superior ao valor (sete) sugerido por Gomes (1990), indicando que as variâncias residuais não foram homogêneas. Por isso, foi realizado o ajuste dos graus de liberdade da interação cultivares com ambientes e do erro combinado, conforme descrito em GoMEs (1990), para a interpretação correta dos testes de hipóteses e para a apresentação adequada da análise de variância conjunta (Tabela 2).

Interação cultivares com ambientes significativa $(\mathrm{P} \leq 0,05)$ foi constatada para a produtividade de grãos de feijão, revelando resposta diferenciada das cultivares às mudanças de ambiente (Tabelas 2 e 3). Por isso, os experimentos de avaliação de cultivares devem ser desenvolvidos em rede, pois diferenças entre locais, anos e épocas de avaliação têm sido observadas em função da diversidade de condições ambientais em que o feijão é cultivado no Brasil (ABREU et al., 1998; CoImBra et al., 1999; NunEs et al., 1999; PiANA et al., 1999; BorGES et al., 2000; CARBONELL et al., 2001; Ribeiro et al., 2004; BACKes et al., 2005; CARBONELl et al., 2007; Melo et al., 2007). Além disso, a pressão de seleção é variável e não previsível. Como conseqüência, a expressão da produtividade de grãos não foi concordante nas diversas condições de cultivo, pois essa é uma característica complexa que envolve mecanismos genéticos, fisiológicos e morfológicos (МеквIв, 2002).

Tabela 2. Análise de variância conjunta da produtividade de grãos $\left(\mathrm{kg} \mathrm{ha}^{-1}\right)$ de 16 cultivares de feijão avaliadas em 20 ambientes no Estado do Rio Grande do Sul, nos anos agrícolas 2004/2005, 2005/2006 e 2006/2007

\begin{tabular}{lccr}
\hline Fonte de variação & GL $\left(^{1}\right)$ & SQ & QM $\left(^{2}\right)$ \\
\hline Cultivar & 15 & 17034804,03 & $1135653,60 \mathrm{~ns}$ \\
Ambiente & 19 & 520915618,70 & $27416611,51^{*}$ \\
Cultivar x Ambiente & $285(145)$ & 111667119,62 & $770118,07^{*}$ \\
Resíduo & $600(298)$ & 64790331,70 & 217417,22 \\
Média $\left(\mathrm{kg} \mathrm{ha}^{-1}\right)$ & 1779 & - & - \\
\hline CV $(\%)$ & 26,21 & - & -
\end{tabular}

${ }^{1}$ ) Valores entre parênteses referem-se aos graus de liberdade ajustados da fonte de variação.

$\left({ }^{2}\right){ }^{*}$ Significativo a $5 \%$ de probabilidade de erro pelo teste F. ${ }^{\text {ns }}=$ não significativo. 
Tabela 3. Médias das cultivares de feijão para a produtividade de grãos de feijão $\left(\mathrm{kg}^{-1}\right)$, coeficiente de variação (CV\%) e índice ambiental (IA) obtidos em 20 ambientes, avaliados nos anos agrícolas 2004/2005, 2005/2006 e 2006/ 2007, em dez locais $\left({ }^{1}\right)$ no Estado do Rio Grande do Sul

\begin{tabular}{|c|c|c|c|c|c|c|c|c|c|c|c|c|c|}
\hline \multirow{2}{*}{ Cultivares } & \multicolumn{5}{|c|}{ (A) } & \multicolumn{3}{|c|}{ (B) } & \multicolumn{2}{|c|}{ (C) } & \multicolumn{3}{|c|}{ (D) } \\
\hline & $2004 / 05$ & & $05 / 06$ & \multicolumn{2}{|c|}{$2006 / 07$} & $2004 / 05$ & $2005 / 06$ & $2006 / 07$ & $2004 / 05$ & $2005 / 06$ & $2004 / 05$ & $2005 / 06$ & $2006 / 07$ \\
\hline Rio Tibagi $\left({ }^{5}\right)$ & $1572 c^{*}$ & & 849 b & \multicolumn{2}{|c|}{$1169 \mathrm{c}$} & $2163 \mathrm{~b}$ & 2229 ns & $621 \mathrm{~b}$ & $2330 \mathrm{~b}$ & $1178 \mathrm{~b}$ & $1444 \mathrm{~d}$ & 2027 a & 3307 a \\
\hline Guateian $6662\left({ }^{5}\right)$ & 1588 c & & $147 \mathrm{~b}$ & \multicolumn{2}{|c|}{837 c } & $2148 \mathrm{~b}$ & 2302 & $1244 \mathrm{~b}$ & 3631 a & $1170 \mathrm{~b}$ & $1766 \mathrm{~b}$ & 2167 a & 3009 a \\
\hline Macanudo $\left({ }^{3}\right)$ & $2163 \mathrm{~b}$ & & 938 a & \multicolumn{2}{|c|}{429 c } & $2160 \mathrm{~b}$ & 2613 & 2196 a & $2718 \mathrm{~b}$ & 1785 a & $1883 \mathrm{~b}$ & $916 \mathrm{~b}$ & 2964 a \\
\hline Minuano $\left({ }^{3}\right)$ & $2013 b$ & & $647 \mathrm{a}$ & \multicolumn{2}{|c|}{$1394 \mathrm{~b}$} & $2030 \mathrm{~b}$ & 2758 & $2174 \mathrm{a}$ & 3843 a & $1406 \mathrm{~b}$ & 2091 a & $1850 \mathrm{a}$ & 1553 a \\
\hline Macotaço $\left({ }^{3}\right)$ & $1685 \mathrm{c}$ & & $742 \mathrm{~b}$ & 107 & $1 \mathrm{c}$ & $2037 \mathrm{~b}$ & 2188 & 2051 a & 4162 a & $1436 \mathrm{~b}$ & $1791 \mathrm{~b}$ & 1818 a & 2549 a \\
\hline IAPAR $44\left({ }^{1}\right)$ & $1163 \mathrm{c}$ & & $623 \mathrm{~b}$ & & $8 \mathrm{c}$ & $2023 \mathrm{~b}$ & 2192 & $532 \mathrm{~b}$ & $1996 \mathrm{~b}$ & $1503 \mathrm{~b}$ & 1617 c & 2472 a & 3003 a \\
\hline Guapo Brilhante $\left({ }^{3}\right)$ & $2305 \mathrm{~b}$ & & 663 a & 197 & 7 a & $2090 \mathrm{~b}$ & 2592 & $1506 \mathrm{~b}$ & $2979 \mathrm{~b}$ & $1361 \mathrm{~b}$ & $1660 \mathrm{c}$ & $2130 \mathrm{a}$ & 3043 a \\
\hline TPS Nobre $\left({ }^{4}\right)$ & $2212 b$ & & 484 a & 236 & 9 a & 2329 a & 2630 & $1150 \mathrm{~b}$ & 3434 a & 1913 a & $1283 \mathrm{e}$ & 2404 a & 2869 a \\
\hline Diamante $\operatorname{Negro}\left({ }^{3}\right)$ & $1710 \mathrm{c}$ & & $135 \mathrm{~b}$ & 141 & $4 \mathrm{~b}$ & $2038 \mathrm{~b}$ & 2361 & 787 b & $2876 \mathrm{~b}$ & $1207 \mathrm{~b}$ & $1151 \mathrm{e}$ & 2136 a & 3073 a \\
\hline BRS Valente $\left({ }^{3}\right)$ & 1994 b & & $211 \mathrm{~b}$ & 144 & $7 \mathrm{~b}$ & $2098 \mathrm{~b}$ & 1899 & $568 \mathrm{~b}$ & $2433 \mathrm{~b}$ & $1321 \mathrm{~b}$ & $1043 \mathrm{f}$ & 1876 a & 3052 a \\
\hline FTS Soberano $\left({ }^{4}\right)$ & $2185 \mathrm{~b}$ & & 761 a & 183 & $0 \mathrm{a}$ & $1920 \mathrm{~b}$ & 2054 & $855 \mathrm{~b}$ & $1813 \mathrm{~b}$ & $1220 \mathrm{~b}$ & $947 \mathrm{f}$ & 2008 a & 2773 a \\
\hline BRS Expedito $\left({ }^{3}\right)$ & $2014 \mathrm{~b}$ & & $115 \mathrm{~b}$ & 144 & $3 \mathrm{~b}$ & 2354 a & 2328 & $914 \mathrm{~b}$ & 3168 a & $1275 \mathrm{~b}$ & $1422 \mathrm{~d}$ & 2164 a & 3262 a \\
\hline Carioca $\left({ }^{2}\right)$ & $1844 \mathrm{c}$ & & $300 \mathrm{~b}$ & 112 & $2 \mathrm{c}$ & 2317 a & 2494 & $1255 \mathrm{~b}$ & 4042 a & 1844 a & $1101 \mathrm{f}$ & 2359 a & 2308 a \\
\hline Iraí $\left({ }^{5}\right)$ & $2644 \mathrm{a}$ & & $275 \mathrm{~b}$ & 159 & $3 \mathrm{~b}$ & 2304 a & 2322 & 2052 a & $2530 \mathrm{~b}$ & $1595 \mathrm{~b}$ & $273 \mathrm{~g}$ & $1461 \mathrm{~b}$ & $2624 \mathrm{a}$ \\
\hline Pérola $\left({ }^{3}\right)$ & $1710 \mathrm{c}$ & & $794 \mathrm{~b}$ & 104 & $0 \mathrm{c}$ & 2304 a & 2059 & $1097 \mathrm{~b}$ & $2454 \mathrm{~b}$ & $1157 \mathrm{~b}$ & $1018 \mathrm{f}$ & 2018 a & 2524 a \\
\hline FTS Magnífico $\left({ }^{4}\right)$ & $1999 \mathrm{~b}$ & & 388 a & & $2 \mathrm{c}$ & $1869 \mathrm{~b}$ & 1603 & $1060 \mathrm{~b}$ & 3252 a & $1544 \mathrm{~b}$ & $1210 \mathrm{e}$ & - & 2647 a \\
\hline TPS Bonito $\left({ }^{4}\right)$ & 2511 a & & 805 a & 181 & 3 a & $1822 \mathrm{~b}$ & 2274 & $1131 \mathrm{~b}$ & 3464 a & 1966 a & $1199 \mathrm{e}$ & $1401 \mathrm{~b}$ & $2723 \mathrm{a}$ \\
\hline IAPAR $31\left(^{1}\right)$ & - & & 800 a & & - & 2468 a & 2859 & $2215 \mathrm{a}$ & $3647 \mathrm{a}$ & 1906 a & $962 \mathrm{f}$ & 2461 a & - \\
\hline TPS Bionobre $\left({ }^{4}\right)$ & $2136 \mathrm{~b}$ & & 515 a & & - & $2430 \mathrm{a}$ & 2631 & $1280 \mathrm{~b}$ & 3728 a & $1462 \mathrm{~b}$ & $1352 \mathrm{e}$ & - & - \\
\hline BRS Campeiro $\left({ }^{3}\right)$ & - & & 671 a & 191 & 3 a & - & 3125 & 1819 a & - & $1332 \mathrm{~b}$ & $1706 \mathrm{c}$ & 1999 a & 3007 a \\
\hline FEPAGRO $26\left({ }^{5}\right)$ & - & & 509 a & & $1 \mathrm{c}$ & - & - & 2046 a & - & - & - & 2262 a & 2873 a \\
\hline Média do ensaio & 1969 & & 351 & 135 & & 2153 & 2376 & 1360 & 3079 & 1479 & 1346 & 1996 & 2798 \\
\hline Média das comuns & 1957 & & 281 & 136 & & 2134 & 2331 & 1258 & 2992 & 1458 & 1356 & 1950 & 2790 \\
\hline CV $(\%)$ & 8,97 & & 17,29 & & 5,11 & 9,37 & 20,84 & 34,24 & 22,66 & 18,89 & 10,35 & 18,46 & 17,13 \\
\hline IA & 178 & & 502 & -41 & & 355 & 552 & -521 & 1213 & -321 & -423 & 171 & 1011 \\
\hline & & & (E) & & & & $(\mathrm{F})$ & $(G)$ & & H) & (I & & $(\mathrm{J})$ \\
\hline Cultivares & $2004 / 20$ & & $2005 / 2$ & 2006 & 2006 & $/ 2007$ & $2004 / 2005$ & $2006 / 2007$ & 2006 & / 2007 & $2004 / 20052$ & $005 / 2006$ & $2006 / 2007$ \\
\hline Rio Tibagi $\left({ }^{5}\right)$ & 944 & & 2422 & 2 a & & 00 a & $978 \mathrm{~b}$ & 1734 a & & 23 a & $229^{n s}$ & $408 \mathrm{c}$ & $2633 \mathrm{~b}$ \\
\hline Guateian $6662\left({ }^{5}\right)$ & 944 & & 1894 & $4 \mathrm{~b}$ & & 58 a & $878 \mathrm{~b}$ & 1722 a & & 83 a & 307 & $692 \mathrm{~b}$ & $3148 \mathrm{~b}$ \\
\hline Macanudo $\left({ }^{3}\right)$ & 967 & & 2167 & 7 a & & 33 a & 1333 a & $1764 \mathrm{a}$ & & 98 a & 329 & $652 \mathrm{~b}$ & $3248 \mathrm{~b}$ \\
\hline Minuano $\left({ }^{3}\right)$ & 1033 & & 2122 & & & 75 a & 1278 a & $1723 \mathrm{a}$ & & $28 \mathrm{a}$ & 333 & $1126 \mathrm{a}$ & 3882 a \\
\hline Macotaço $\left({ }^{3}\right)$ & 1100 & & 2100 & 0 a & & 83 a & 1356 a & $1524 \mathrm{~b}$ & & $50 \mathrm{a}$ & 444 & 807 b & $3043 \mathrm{~b}$ \\
\hline IAPAR $44\left({ }^{1}\right)$ & 1189 & & 1833 & $3 \mathrm{~b}$ & & 08 a & $711 \mathrm{c}$ & $1857 \mathrm{a}$ & & $30 \mathrm{a}$ & 277 & $476 \mathrm{c}$ & 3663 a \\
\hline Guapo Brilhante $\left({ }^{3}\right)$ & 967 & & 1822 & $2 b$ & & 17 a & $1244 \mathrm{a}$ & $1518 \mathrm{~b}$ & & $58 \mathrm{~b}$ & 223 & $872 \mathrm{~b}$ & 2913 b \\
\hline TPS Nobre $\left({ }^{4}\right)$ & 1189 & & 2194 & 4 a & & $00 \mathrm{a}$ & 1433 a & $1561 \mathrm{~b}$ & & 77 b & 250 & $856 \mathrm{~b}$ & 3562 a \\
\hline Diamante Negro $\left({ }^{3}\right)$ & 911 & & 2156 & 6 a & & $17 \mathrm{~b}$ & 1156 a & $1549 \mathrm{~b}$ & & 05 a & 342 & $338 c$ & $3033 \mathrm{~b}$ \\
\hline BRS Valente $\left({ }^{3}\right)$ & 978 & & 2356 & 6 a & & $08 \mathrm{~b}$ & $1144 \mathrm{a}$ & 1772 & & $75 \mathrm{~b}$ & 269 & $440 \mathrm{c}$ & $2997 \mathrm{~b}$ \\
\hline FTS Soberano $\left({ }^{4}\right)$ & 1089 & & 1467 & & & $92 \mathrm{~b}$ & $1056 \mathrm{~b}$ & 1769 a & & $23 \mathrm{~b}$ & 253 & $705 \mathrm{~b}$ & 2952 b \\
\hline BRS Expedito $\left({ }^{3}\right)$ & 1011 & & 2211 & 1 a & & 08 a & 1556 a & 1792 a & & $10 \mathrm{a}$ & 227 & $421 \mathrm{c}$ & 3658 a \\
\hline Carioca $\left({ }^{2}\right)$ & 1156 & & 2233 & 3 a & & $58 \mathrm{~b}$ & 1422 a & $1644 \mathrm{a}$ & & $08 \mathrm{~b}$ & 513 & $821 \mathrm{~b}$ & 3692 a \\
\hline Iraí $\left({ }^{5}\right)$ & 833 & & 2122 & 2 a & & $50 \mathrm{~b}$ & $422 \mathrm{c}$ & $1357 \mathrm{~b}$ & & $360 \mathrm{c}$ & 212 & $483 \mathrm{c}$ & $2518 \mathrm{~b}$ \\
\hline Pérola $\left({ }^{3}\right)$ & 633 & & 1722 & $2 \mathrm{~b}$ & & 58 a & 1289 a & $1408 \mathrm{~b}$ & & $07 \mathrm{~b}$ & 340 & 417 & 3566 a \\
\hline FTS Magnífico $\left({ }^{4}\right)$ & 1022 & & & - & & $58 \mathrm{~b}$ & $989 \mathrm{~b}$ & $1331 \mathrm{~b}$ & & $75 \mathrm{~b}$ & 398 & - & $3223 \mathrm{~b}$ \\
\hline TPS Bonito $\left({ }^{4}\right)$ & 1144 & & 2233 & 3 a & & $67 \mathrm{~b}$ & 1178 a & $1479 \mathrm{~b}$ & & 63 a & 274 & $213 c$ & 3353 a \\
\hline IAPAR $31\left({ }^{1}\right)$ & - & & 2344 & $4 \mathrm{a}$ & & - & - & - & & - & - & 974 a & - \\
\hline TPS Bionobre $\left({ }^{4}\right)$ & - & & & - & & - & - & - & & - & - & - & - \\
\hline BRS Campeiro $\left({ }^{3}\right)$ & - & & 1944 & $4 \mathrm{~b}$ & & 58 a & - & $1622 \mathrm{a}$ & & $23 \mathrm{a}$ & - & $563 c$ & 3339 a \\
\hline FEPAGRO $26\left({ }^{5}\right)$ & - & & 2067 & 7 a & & $08 \mathrm{~b}$ & - & $1701 \mathrm{a}$ & & $51 \mathrm{~b}$ & - & $834 \mathrm{~b}$ & 3437 a \\
\hline Média & 1007 & & 2074 & & & 98 & 1142 & 1623 & & 66 & 307 & 637 & 3256 \\
\hline Média das comuns & 1006 & & 2066 & & & 27 & 1152 & 1636 & & 69 & 301 & 608 & 3241 \\
\hline CV $(\%)$ & & 62 & & 8,55 & & 14,98 & 16,02 & 9,55 & & 12,26 & 39,97 & 21,69 & 13,11 \\
\hline IA & -773 & & 287 & & & 52 & -627 & -143 & & 90 & -1478 & -1171 & 1462 \\
\hline
\end{tabular}

$\left({ }^{1}\right)$ Locais: (A) Santa Maria; (B) Pelotas; (C) Frederico Westphalen; (D) Maquiné; (E) Santo Augusto; (F) São Borja; (G) Palmeira das Missões; (H) Vacaria; (I) Júlio de Castilhos; (J) Veranópolis.

$\left({ }^{2}\right)$ Obtentor/Detentor da cultivar de acordo com MAPA (2007): $\left({ }^{1}\right)$ Instituto Agronômico do Paraná (IAPAR); $\left({ }^{2}\right)$ Instituto Agronômico (IAC); de Campinas; $\left({ }^{3}\right)$ Empresa Brasileira de Pesquisa Agropecuária (EMBRAPA); $\left({ }^{4}\right)$ Francisco Terassawa - Pesquisa e Sementes (FT - Sementes); ${ }^{(5)}$ Fundação Estadual de Pesquisa Agropecuária (FEPAGRO).

${ }^{*}$ Médias seguidas por letras iguais não diferem entre si pelo teste de Scott-Knott, a 5\% de probabilidade de erro. ${ }^{\text {ns }}$ Não significativo. 
A média obtida para a produtividade de grãos nos experimentos de Santa Maria (2004 / 2005 e 2005/ 2006), Pelotas (2004/2005 e 2005/2006), Frederico Westphalen (2004/2005), Maquiné (2005/2006 e 2006/2007), Santo Augusto (2005/2006), Vacaria (2006/2007) e Veranópolis (2006/2007) foi superior à média geral $\left(1779 \mathrm{~kg} \mathrm{ha}^{-1}\right)$, caracterizando ambientes favoráveis (índices ambientais positivos) (Tabela 3). Nos demais experimentos os índices ambientais foram negativos, representando ambientes desfavoráveis. Assim, será possível a identificação de cultivares com adaptação ampla e específica e verificar sua previsibilidade de comportamento.

A avaliação das cultivares pelo método de Eberhart e RusSell (1966) permitiu identificar a cultivar FTS Soberano com adaptação específica a ambientes desfavoráveis $\left(\beta_{\mathrm{i}}<1\right)$ (Tabela 4$)$. Por sua vez, as cultivares BRS Expedito e TPS Bonito são adaptadas a ambientes favoráveis $\left(\beta_{\mathrm{i}}>1\right)$. As demais cultivares foram de adaptação geral às condições de cultivo do Estado do Rio Grande do Sul, pois o coeficiente $b_{i}$ foi semelhante à unidade $\left(\beta_{i}=1\right)$. Assim, as cultivares mais adaptadas e produtivas para tipo de grão comercial preto foram TPS Nobre, Minuano, Macanudo, Guapo Brilhante, Macotaço, BRS Expedito e Guateian 6662; e para tipo de grão comercial carioca foram Carioca e TPS Bonito.

As cultivares TPS Nobre e Minuano podem ser consideradas promissoras, pois além das maiores médias para a produtividade de grãos, de $1985 \mathrm{~kg} \mathrm{ha}^{-1}$ e $1983 \mathrm{~kg} \mathrm{ha}^{-1}$ respectivamente, possuem ampla adaptabilidade. No entanto, a baixa previsibilidade de comportamento $\left(\mathrm{s}^{2} \mathrm{~d}_{\mathrm{i}} \# 0\right)$, observada nessas cultivares, constitui uma desvantagem. Como os valores obtidos de $R^{2}$ foram considerados altos, a TPS Nobre e a Minuano não deverão ser julgadas como indesejáveis para o cultivo no Rio Grande do Sul.

Tabela 4. Produtividade média de grãos (prod., $\mathrm{kg} \mathrm{ha}^{-1}$ ) de 16 cultivares de feijão e estimativas dos parâmetros de adaptabilidade e de estabilidade obtidos pelos métodos de EBERHART e Russel (1966) - coeficiente de regressão $\left(\beta_{\mathrm{i}}\right)$, desvio de regressão $\left(\right.$ ó $\left.^{2} \mathrm{~d}_{\mathrm{i}}\right)$ e coeficiente de determinação $\left(\mathrm{R}^{2}, \%\right)$, e de LIN e BINSS (1988), modificado por CARNEIRO (1998) - índice $\mathrm{P}_{\mathrm{i}}\left(\mathrm{P}_{\mathrm{i}}\right)$, adaptação a ambiente favorável $\left(\mathrm{P}_{\mathrm{if}}\right)$ e a ambiente desfavorável ( $\left.\mathrm{P}_{\mathrm{id}}\right)$, avaliados em 20 ambientes no Estado do Rio Grande do Sul

\begin{tabular}{|c|c|c|c|c|c|c|c|}
\hline Cultivar & Rend. & $\beta_{\mathrm{i}}^{1}$ & $\mathrm{o}^{2} \mathrm{di}^{2}$ & $\mathrm{R} 2$ & $\mathrm{P}_{\mathrm{i}}$ & $P_{\text {if }}$ & $\mathrm{P}_{\mathrm{id}}$ \\
\hline & & & & $\%$ & 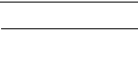 & $-10^{4}$ & \\
\hline TPS Nobre & 1985 & $1,0441^{\mathrm{ns}}$ & $42643,37^{*}$ & 89,31 & 10,4142 & 10,3825 & 10,4458 \\
\hline Minuano & 1983 & $0,9726^{\mathrm{ns}}$ & $196088,61^{*}$ & 71,07 & 14,4248 & 21,2141 & 7,6355 \\
\hline Macanudo & 1888 & $0,9702^{\text {ns }}$ & $188490,97^{*}$ & 71,65 & 24,0506 & 27,0576 & 21,0437 \\
\hline Guapo Brilhante & 1882 & $0,9409^{\text {ns }}$ & $21496,4^{\mathrm{ns}}$ & 90,28 & 13,3689 & 18,3607 & 8,3770 \\
\hline Carioca & 1882 & $1,0799 \mathrm{~ns}$ & $83061,35^{*}$ & 85,52 & 17,8514 & 15,6353 & 20,0674 \\
\hline Macotaço & 1867 & $0,9449^{\text {ns }}$ & $130276,47^{*}$ & 76,40 & 17,4714 & 23,1553 & 11,7876 \\
\hline BRS Expedito & 1858 & $1,1484^{*}$ & $-2119,49^{\mathrm{ns}}$ & 95,91 & 17,3630 & 13,7313 & 20,9947 \\
\hline TPS Bonito & 1851 & $1,1145^{*}$ & $82098,94^{*}$ & 86,38 & 17,0264 & 14,2169 & 19,8360 \\
\hline Guateian 6662 & 1792 & $1,0886^{\mathrm{ns}}$ & $41211,93^{*}$ & 90,25 & 20,4989 & 17,0885 & 23,9094 \\
\hline Diamante Negro & 1700 & $1,0563^{\mathrm{ns}}$ & $1968,22^{\mathrm{ns}}$ & 94,66 & 26,2061 & 22,5939 & 29,8182 \\
\hline IAPAR 44 & 1675 & $0,9805^{\mathrm{ns}}$ & $175120,49^{*}$ & 73,30 & 40,2983 & 48,2351 & 32,3615 \\
\hline Rio Tibagi & 1658 & $0,9630^{\mathrm{ns}}$ & $69387,73^{*}$ & 84,14 & 35,2248 & 39,7981 & 30,6516 \\
\hline BRS Valente & 1639 & $0,9746^{\mathrm{ns}}$ & $36407,82^{*}$ & 88,78 & 33,7540 & 34,6519 & 32,8561 \\
\hline FTS Soberano & 1633 & $0,8318^{*}$ & $96766,86^{*}$ & 75,86 & 36,8562 & 48,0398 & 25,6726 \\
\hline Pérola & 1606 & $0,9905^{\mathrm{ns}}$ & $19579,96^{\mathrm{ns}}$ & 91,41 & 34,1658 & 37,8602 & 30,4713 \\
\hline Iraí & 1566 & $0,8992^{\mathrm{ns}}$ & $247060,52^{*}$ & 63,27 & 44,5004 & 49,9396 & 39,0613 \\
\hline
\end{tabular}

Média geral

1779

$\left({ }^{1}\right) \mathrm{H} 0=\beta_{\mathrm{i}}=1 *$ significativo a $5 \%$ de probabilidade pelo teste $\mathrm{t}$; ns: não significativo.

( $\left.{ }^{2}\right) \mathrm{H}_{0}=\mathrm{o}^{2} \mathrm{~d}_{\mathrm{i}}=0$ * significativo a $5 \%$ de probabilidade pelo teste $\mathrm{F}$; ns: não significativo. 
As cultivares Guapo Brilhante, Diamante Negro e Pérola foram estáveis (desvio da regressão não significativo). Entretanto, as cultivares Diamante Negro e Pérola obtiveram produtividade média inferior à média geral. Assim, apenas a cultivar Guapo Brilhante foi considerada desejável, segundo os critérios de Eberhart e Russel (1666), ou seja, com ampla adaptabilidade (coeficiente de regressão igual a unidade), estabilidade elevada (desvio de regressão igual a zero) e produtividade de grãos superior à média geral $\left(1882 \mathrm{~kg} \mathrm{ha}^{-1}\right)$. Por sua vez, PIANA et al. (1999), não identificaram nenhuma cultivar com estabilidade de produção para o Estado do Rio Grande do Sul, quando avaliaram 11 genótipos de feijão, em 72 ambientes de cultivo, durante o período de 1988 a 1994. Dessa maneira, o desenvolvimento de germoplasma de feijão com alto potencial de produtividade de grãos, adaptação e estabilidade de produção foi possível nesse Estado, comprovando a eficiência na seleção dos programas de melhoramento.

Pelo método de LiN e BINNS (1988), as cultivares com menor valor de $P_{i}$ foram TPS Nobre, Minuano e Guapo Brilhante, evidenciando maior estabilidade de produção, além de alta produtividade média nos ambientes considerados (Tabela 4). As cultivares com menor $P_{i}$ são desejáveis, pois obtiveram menor desvio em relação à produtividade máxima em cada ambiente, ou seja, desempenho próximo ao máximo na maioria dos ambientes. A metodologia de Lin e Binns (1988) também possibilitou a identificação de cultivares e de linhagens de feijão com alta estabilidade fenotípica associada ao alto desempenho médio em vários ambientes de cultivo (ABREU et al., 1998; NunEs et al., 1999; Borges et al., 2000; CARBONELL et al., 2001; BACKES et al., 2005; CARbonell et al., 2007; Melo et al., 2007).

Considerando a decomposição proposta por CARneIro (1998), as cultivares TPS Nobre, BRS Expedito e TPS Bonito foram de adaptação a ambientes favoráveis $\left(<\mathrm{P}_{\text {if }}\right)$ e as cultivares TPS Nobre, Minuano, Guapo Brilhante e Macotaço foram adaptadas a ambientes desfavoráveis $\left(<\mathrm{P}_{\mathrm{id}}\right)$ (Tabela 4). De maneira semelhante, CARbonell et al. (2001) e Melo et al. (2007) também identificaram germoplasma de feijão com maior estabilidade, mais responsivos a ambientes favoráveis e mais adaptados a ambientes desfavoráveis, utilizando a mesma decomposição.

A cultivar TPS Nobre possui estabilidade de produção (menor $\mathrm{P}_{\mathrm{i}}$ ), adaptação a ambientes favoráveis $\left(<\mathrm{P}_{\mathrm{if}}\right)$ e a ambientes desfavoráveis $\left(<\mathrm{P}_{\mathrm{id}}\right)$, segundo o método de LiN e BinNs (1988), modificado por CARNEIRO (1998), bem como ampla adaptabilidade pelo método de EBERHART e Russel (1966) e a maior produtividade média de grãos $\left(1985 \mathrm{~kg} \mathrm{ha}^{-1}\right)$ nos 20 ambientes. Essa cultivar não está registrada para o cultivo no Rio Grande do Sul, entretanto, como é de excelente potencial de produção e estabilidade, sua inclusão no RNC-MAPA deve ser considerada pelo obtentor da cultivar.

As metodologias de EberHART e Russell (1966) e de Lin e BINNs (1988), modificada por CARNEIRO (1998) foram concordantes na identificação de cultivares com estabilidade de produção (Guapo Brilhante) e com adaptação a ambientes favoráveis (BRS Expedito e TPS Bonito). Assim, as cultivares da Embrapa (Guapo Brilhante e BRS Expedito) constituem boas opções de cultivo, pois possuem grãos de tegumento preto que são aqueles de maior preferência para o consumo e que têm maior volume de comercialização no Estado do Rio Grande do Sul. A cultivar Guapo Brilhante já é recomendada há muitos anos para o cultivo no Estado e a cultivar BRS Expedito foi, recentemente, inscrita no RNC-MAPA. A cultivar TPS Bonito é uma alternativa para expansão da área cultivada com grãos do tipo carioca no RS e sua inscrição no MAPA pode ser requerida pelo obtentor da cultivar.

\section{CONCLUSÃO}

A cultivar Guapo Brilhante é de alta produtividade média de grãos, com adaptação e estabilidade de produção para o cultivo no Estado do Rio Grande do Sul, de acordo com os métodos de Eberhart e Russel e de Lins e Binns, modificado por Carneiro.

\section{AGRADECIMENTOS}

Ao Conselho Nacional de Desenvolvimento Científico e Tecnológico (CNPq) pela bolsa concedida. Aos colegas Guido Renato Sander, Antônio Losso, Lineu Migon, Evandro Missio e Nilton Gabe da FEPAGRO, pelo auxílio na realização dos experimentos.

\section{REFERÊNCIAS}

ABREU, A.F.B.; RAMALHO, M.A.P.; ANDRADE, M.J.B.; PEREIRA FILHO, I.A. Estabilidade de linhagens de feijão em algumas localidades do Estado de Minas Gerais no período de 1994 a 1995. Ciência e Agrotecnologia, Lavras, v.22, n.3, p.308312, 1998.

ANTUNES, P.L.; BIHALVA, A.B.; ELIAS, M.C.; SOARES, G.J.D. Valor nutricional de feijão (Phaseolus vulgaris L.), cultivares Rico 23, Carioca, Piratã-1 e Rosinha-G2. Revista Brasileira de Agrociência, Pelotas, v.1, n.1, p.12-18, 1995. 
BACKES, R.L.; ELIAS, H.T.; HEMP, S.; NICKNICH, W. Adaptabilidade e estabilidade de genótipos de feijoeiro no Estado de Santa Catarina. Acta Scientiarum Agronomy, Maringá, v.27, n.2, p.309-314, 2005.

BORGES, L.C.; FERREIRA, D.F.; ABREU, A.F.B.; RAMALHO, M.A.P. Emprego de metodologias de avaliação da estabilidade fenotípica na cultura do feijoeiro (Phaseolus vulgaris L.). Revista Ceres, Viçosa, v.47, n.269, p.89-102, 2000.

BRASIL. Ministério da Agricultura, Pecuária e Abastecimento. Anexo IV. Requisitos mínimos para determinação do valor de cultivo e uso de feijão (Phaseolus vulgaris), para a inscrição no registro nacional de cultivares - RCN. Disponível em: http:// www.agricultura.gov.br. Acesso em 21 ago. 2006.

CARNEIRO, P.C.S. Novas metodologias de análise da adaptabilidade e estabilidade de comportamento. 1998. 155f. Tese (Doutorado em Genética e Melhoramento) Universidade Federal de Viçosa, Viçosa, 1998.

CARBONELL, S.A.M.; AZEVEDO FILHO, J.A.; DIAS, L.A.S; GONÇALVES, C.; ANTONIO, C.B. Adaptabilidade e estabilidade de produção de cultivares e linhagens de feijoeiro no Estado de São Paulo. Bragantia, Campinas, v.60, n.2, p.6977, 2001.

CARBONELL, S.A.M.; CHIORATO, A.F.; RESENDE, M.D.V.; DIAS, L.A.S.; BERALDO, A.L.A.; PERINA, E.F. Estabilidade de cultivares e linhagens de feijoeiro em diferentes ambientes no Estado de São Paulo. Bragantia, Campinas, v.66, n.2, p.193201, 2007.

CEPEF. Indicações técnicas para a cultura do feijão no Rio Grande do Sul. Passo Fundo: UPF, 2003. 149p.

COIMBRA, J.L.M.; CARVALHO, F.I.F.; HEMP, S.; SILVA, S.A. Adaptabilidade e estabilidade fenotípica em genótipos de feijão de cor (Phaseolus vulgaris.L.) em três ambientes distintos. Ciência Rural, Santa Maria, v.29, n.3, p.441-448, 1999.

CONAB - Companhia Nacional de Abastecimento. Acompanhamento da safra brasileira: grãos: $12^{\circ}$ levantamento, setembro de 2007. Brasília: CONAB, 2007. 24p. Disponível em: http:// www.conab.gov.br. Acesso em 25 jan. 2008.

CRUZ, C.D.; REGAZZI, A.J. Modelos biométricos aplicados ao melhoramento genético. 2.ed. Viçosa:UFV, 2001. 390p.

CRUZ, C.D. Programa Genes: biometria. Viçosa: UFV, 2006. 382p.

CRUZ, C. D.; CARNEIRO, P. C. S. Modelos biométricos aplicados ao melhoramento genético. Volume 2. Viçosa: Editora UFV, 2003. 585p.

EBERHART, S.A.; RUSSELL, W.A. Stability parameters for comparing varieties. Crop Science, Stanford, v.6, n.1, p.3640, 1966

GOMES, F.P. Curso de estatística experimental. 13. ed. Piracicaba: São Paulo: Nobel 1990. 468 p.
HOSFIELD, G.L. Genetic control of production and food quality factors in dry bean. Food Technology, Chicago, v.45, n.9, p.98103, 1991.

LIN, C.S.; BINNS, M.R. Superiority measure of cultivar performance for cultivar $x$ location data. Canadian Journal of Plant Science, Ottawa, v.68, p.193-198, 1988.

MAPA - Ministério da Agricultura, Pecuária e Abastecimento. Zoneamento agrícola de risco climatico: cultivares de feijão ano - safra 2006/2007. Brasília: MAPA, 2007. 14p. Disponível em: http:/ /www.agricultura.gov.br. Acesso em 11 dez. 2008.

MEKBIB, F. Simultaneous selection for high yield and stability in common bean (Phaseolus vulgaris L.) genotypes. Journal of Agricultural Science, Cambridge, v.138, n.3, p.249-253, 2002.

MELO, L.C.; MELO, P.G.S.; FARIA, L.C.; DIAZ, J.L.C.; DEL PELOSO, M.J.; RAVA, C.A.; COSTA, J.G.C. Interação com ambientes e estabilidade de genótipos de feijoeiro- comum na Região Centro-Sul do Brasil. Pesquisa Agropecuária Brasileira, Brasília, v.42, n.5, p.715-723, 2007.

MORROW, B. The rebirth of legumes. Food Technology, Chicago, v.45, n.9, p.96- 121, 1991.

NUNES, G.H.S. Estabilidade de cultivares de feijão-comum no Estado de Santa Catarina. Revista Ceres, Viçosa, v.46, n.268, p.625-633, 1999.

PIANA, C.F.B.; ANTUNES, I.F.; SILVA, J.G.C.; SILVEIRA, E.P. Adaptabilidade e estabilidade do rendimento de grãos de genótipos de feijão. Pesquisa Agropecuária Brasileira, Brasília, v.34, n.4, p.553-564, 1999.

RIBEIRO, N.D.; JOST, E.; POSSEBON, S.B.; CARGNELUTTI FILHO, A. Adaptabilidade e estabilidade de cultivares registradas de feijão em diferentes épocas de semeadura para a depressão central do Rio Grande do Sul. Ciência Rural, Santa Maria, v.34, n.5, p.1395-1400, 2004.

SILVA, W.C.J.; DUARTE, J.B. Métodos estatísticos para estudo de adaptabilidade e estabilidade fenotípica em soja. Pesquisa Agropecuária Brasileira, Brasília, v.41, n.1, p.23-30, 2006.

STEEL, R.G.D. et al. Principles and procedures of statistics a biometrical approach. 3.ed. New York: McGraw Hill Book, 1997. 666p. 